TITLE:

\title{
Surface plasmon field-enhanced fluorescence spectroscopy apparatus with a convergent optical system for point-of-care testing.
}

\section{AUTHOR(S):}

Toda, Mitsuaki; Arima, Yusuke; Takiguchi, Hiromi; Iwata, Hiroo

\section{CITATION:}

Toda, Mitsuaki ...[et al]. Surface plasmon field-enhanced fluorescence spectroscopy apparatus with a convergent optical system for point-of-care testing.. Analytical biochemistry 2014, 467(15): 47-53

ISSUE DATE:

2014-09-06

URL:

http://hdl.handle.net/2433/191329

\section{RIGHT:}

(C) 2014 Elsevier Inc:; This is not the published version. Please cite only the published version.; この論文は出版社版でありません。引用の際に は出版社版をご確認ご利用ください。 
Immunological Procedures

Surface plasmon field-enhanced fluorescence spectroscopy apparatus with a convergent optical system for point-of-care testing

\author{
Mitsuaki TODA ${ }^{\mathrm{a}, \dagger}$, Yusuke ARIMA ${ }^{\mathrm{a}}$, Hiromi TAKIGUCHI ${ }^{\mathrm{b}}$ and Hiroo IWATA ${ }^{\mathrm{a}, *}$ \\ ${ }^{a}$ Institute for Frontier Medical Sciences, Kyoto University, \\ 53 Kawahara-cho, Shogoin, Sakyo-ku, Kyoto 606-8507, Japan \\ ${ }^{\mathrm{b}}$ Graduate School of Engineering, The University of Tokyo, \\ 7-3-1, Hongo, Tokyo, 113-8656, Japan.
}

Short title: SPFS apparatus with a convergent optical system

*Corresponding address:

Professor Dr. Hiroo IWATA

Department of Reparative Materials, Field of Tissue Engineering,

Institute for Frontier Medical Sciences, Kyoto University.

53 Kawahara-cho, Shogoin, Sakyo-ku, Kyoto 606-8507, Japan.

Phone/FAX: +81-75-751-4119

E-mail: iwata@frontier.kyoto-u.ac.jp

${ }^{\dagger}$ Present address: Office of Society-Academia Collaboration for Innovation, Kyoto University. Yoshida-honnmachi, Sakyo-ku, Kyoto 606-8501, Japan. 


\section{Abstract}

Surface plasmon field-enhanced fluorescence spectroscopy (SPFS) is a promising methodology for point-of-care (POC) testing. The SPFS devices which have been reported have been equipped with an angle rotating stage to adjust the surface plasmon resonance (SPR) angle. In a clinical setting, however, the SPR angle determination is a tedious and time consuming process. In this study, we employed an SPFS instrument with a convergent optical system that allows the omission of this procedure. We demonstrated that this instrumentation allowed the sensitive determination of low concentrations of alpha-fetoprotein in serum and reduced the variation effect caused by the protein concentrations in samples. The SPFS with a convergent optical system is suitable for POC testing.

Key Word: Surface plasmon-field enhanced fluorescence spectroscopy; Convergent optical system; Tumor Marker; Highly sensitive detection 


\section{Introduction}

Point-of-care $(\mathrm{POC})^{1}$ testing allows medical personnel to carry out clinical tests at

the bed side or near patients [1]. The results are immediately available, thus permitting the rapid application of clinical management decisions. Various methods such as paper chromatography [2], electrochemical analysis [3], surface plasmon resonance [4-7], and so on $[8,9]$ have been examined to develop point-of-care testing (POCT) devices. Surface plasmon field-enhanced fluorescence spectroscopy (SPFS) [10] (Scheme 1) is a promising method for the development of a highly sensitive POC device. SPFS utilizes a field-enhanced optical field of a surface plasmon mode for the excitation of fluorophores placed near the metal-dielectric interface. The evanescent field of a surface plasmon is enhanced by a factor of about 15 compared to the incident field for a gold-water interface at the specific resonance angle and then the signal decays exponentially into the dielectric medium, approximately Lz $=190 \mathrm{~nm}$ [11]. Thus, an SPFS-based sensor allows the sensitive determination of low concentrations of target molecules without the removal of unbounded complexes or $\mathrm{B} / \mathrm{F}$ separation steps. Yu et al. reported a SPFS-based sensing device and achieved highly sensitive detection of free prostate specific antigen (f-PSA) [12]. Our group also developed an SPFS-based device and demonstrated quantitative detection of alpha-fetoprotein (AFP), a marker of hepatoma, at levels below the cut-off concentration for the clinical test [13]. Some problems, however, remain for the SPFS-based sensing devices used for POCT. Protein 
concentration in patients' blood varies within the range of $30-100 \mathrm{mg} / \mathrm{mL}[14,15]$, resulting in large variation of its refractive indices. Conventional SPFS devices monitor a change in fluorescence intensity at a fixed incident angle near the surface plasmon resonance (SPR) angle, which is influenced by the refractive index near the metal-dielectric interface. Therefore, the incident angle must be carefully adjusted to be close to the SPR angle for each patient to maximize the field intensity of the surface plasmon and thus the fluorescence intensity.

The SPFS-based devices, which have been previously reported, are equipped with an angle rotating stage to adjust the SPR angle $[12,13]$. These devices should be small and consist of simple optical units for their application to POCT. In this study, we developed an SPFS-based device with a convergent optical system. Parallel incident light was focused through the lenses and a prism onto the back side of sensor chip surface, giving a fixed range of incident light angles. We examined the SPFS-based device with a convergent optical system for detecting AFP included in different concentrations of bovine serum albumin (BSA).

\section{Materials and methods}

Materials, reagents and antibodies

11-Mercaptoundecanoic acid (Sigma-Aldrich Co., St. Louis, MO, USA), 
1-ethyl-3-(3-dimethylaminopropyl) carbodiimide hydrochloride (EDC, Dojindo, Kumamoto, Japan), ethanol and $N$-hydroxysuccinimide (Nacalai Tesque, Kyoto Japan) and polyoxyethylene sorbitan monolaurate (Tween 20(R) equivalent, Wako Pure Chemical Industry, Osaka, Japan) were of reagent grade and used as obtained. Dulbecco’s PBS powder (D-PBS, Nissui Pharmaceuticals, Tokyo, Japan), bovine serum albumin (BSA, Sigma-Aldrich, USA), and alpha-fetoprotein (AFP, HyTest, Turku, Finland) were used as obtained. Fetal bovine serum (FBS, Biowest SAS, Nuaille, France) was heated at $56{ }^{\circ} \mathrm{C}$ to inactivate the complement system before use. Water was purified with a MilliQ system (Millipore Co.). Monoclonal mouse anti-human AFP antibody (clone 1D5 and 6D2) was obtained from Mikuri Immunological Laboratories (Osaka, Japan) and its solution was prepared and stored in accordance with supplier instructions. Anti-human AFP (clone 6D2) was labeled by fluorescence dye (Alexa-Fluor(R) 647) following the supplier's instruction for the labeling kit (Invitrogen ${ }^{\mathrm{TM}}$, Life Technologies, Co., Carlsbad, CA, USA.). Concentration of Alexa-Fluor 647 labeled anti-human AFP (clone 6D2) in the stock solution was determined by the method presented in the instruction from the absorbance at $280 \mathrm{~nm}$ and $650 \mathrm{~nm}$ and molar absorptivity $\left(\varepsilon_{\mathrm{Ab}}=203,000\right)$ of antibodies.

Preparation of SPFS apparatus with convergent optical system

The SPFS apparatus with a convergent optical system (referred to as 
SPFS-convergent) prepared in this study is schematically shown in Fig. 2(a). A $650 \mathrm{~nm}$ light source of pointed LED (BL15-1212, Kodenshi corp., Kyoto, Japan) was collimated using an aspheric optical lens (No.65989, Edmond Optics Japan, Ltd, Tokyo, Japan). The light was passed through an iris (IH-08R, Sigma Koki, Tokyo, Japan) to cut the light of which the directivity was larger than 5 degrees and then linearly p-polarized with an optical filter (NT47-215, Edmond Optics Japan, Ltd, Tokyo, Japan). An S-LAL10 glass plate coated with a chromium underlayer $(1 \mathrm{~nm})$ and a thin gold layer $(49 \mathrm{~nm})$ was optically coupled to a hemi-cylinder prism via immersion oil (ref. index: 1.720, Cargille Laboratories Inc., NJ, USA). A flow cell was assembled on the glass plate by fixing a poly(methylmethacrylate) (PMMA) plate through a spacer made of silicone rubber (thickness: $1 \mathrm{~mm}$ ), and silicone tubes (inner diameter: $0.5 \mathrm{~mm}$, outer diameter: $1 \mathrm{~mm}$ ) were connected to the space on the glass plate through the PMMA plate [13]. The sample solution or D-PBS was delivered to the glass plate through the flow cell with a peristaltic pump (SMP-21, Tokyo Rikakikai Co., Ltd., Tokyo, Japan). The p-polarized and collimated light was focused on the gold layer by an aspheric optical lens (No.48164, Edmond Optics Japan, Ltd, Tokyo, Japan).

The reflected light was captured by a CCD (ICX086AK; image sensor for NTSC color video cameras, Sony Corp., Japan) camera module (without cooling, outputting NTSC signals) and its image was recorded by a PC with recording software (VirtualDubMod ver. 1.4.13.1jp2) and analyzed by ImageJ (ver.1.4.3). The fluorescence image on the surface of the 
glass plate was collected through an objective lens (SLWD Plan20×, Nikon, Tokyo, Japan) and $670 \mathrm{~nm}$ interference filter (Optical Coatings Japan, Tokyo, Japan) with a high-sensitivity EM-CCD camera equipped with a charge multiplier (MC681-SPD; Texas Instruments, Dallas, TX, USA) cooled by a built-in peltier cooler. Acquired images were captured with an image capture board (MT-PCI2; Micro-Technica, Tokyo, Japan) and analyzed with home-made intensity scanning software. The fluorescence light intensities in selected areas $(100 \times 100$ pixels, corresponding to $210 \mu \mathrm{m} \times 210 \mu \mathrm{m}$ ) were determined.

Preparation of SPFS apparatus with angle rotation stage

The SPFS instrument of Kretschmann configuration was fabricated in-house (referred to as SPFS-1spot) [13], following the setup described by Knoll [10], with minor modifications. Briefly, a glass plate with a thin gold layer was coupled to a triangular prism via immersion oil (Cargille). A flow cell was prepared as mentioned above. A laser diode (Coherent, Santa Clara, CA, USA) was used as a source of incident light ( $\lambda=635 \mathrm{~nm}, 0.95$ $\mathrm{mW}$ ). The laser intensity was reduced to $10 \%$ with a neutral density (ND) filter. The laser was linearly p-polarized, then irradiated through a triangular prism at the back side of the glass plate. The incident angle was kept constant during fluorescence detection. The reflected light intensity was monitored with a photodiode detector (S2281-04; Hamamatsu Photonics, Hamamatsu, Japan). The fluorescence image on the surface of the glass plate was collected 
by a CCD camera (MC681-SPD) and analyzed as mentioned above.

Immobilization of antibodies on a sensor chip

Glass plates (S-LAL10, refractive index: $1.720,25 \times 25 \times 1 \mathrm{~mm}$ ) were purchased from

Sigma Koki (Tokyo, Japan). They were coated with a gold layer as previously reported [13,

15]. Briefly, S-LAL10 glass plates were cleaned by plasma treatment in a plasma reactor

(PA300AT; O-kuma Engineering Fukuoka, Japan) under $5 \mathrm{~Pa}_{2}$ for 1 min., rinsed with deionized water and 2-propanol (Nacalai Tesque) three times each and then dried under a stream of nitrogen gas (Kyoto Teisan, Kyoto Japan). The glass plates were coated with $1 \mathrm{~nm}$ chromium and then with gold, $49 \mathrm{~nm}$ in thickness, by a thermal evaporation apparatus (V-KS200, Osaka Vacuum Ltd., Osaka, Japan). 11-Mercaptoundecanoic acid was dissolved in ethanol deoxygenized by bubbling of nitrogen gas. The gold-coated glass plate was immersed in a $1 \mathrm{mM}$ solution of 11-mercaptoundecanoic acid at room temperature for at least $24 \mathrm{~h}$ to form a self-assembled monolayer (SAM) of the carboxy-terminated alkanethiol. Then, the glass plate was sequentially washed with ethanol and Milli-Q water three times each and then dried under a stream of dried nitrogen gas. A piece of silicone rubber sheet (Togawa Rubber Co., Ltd., Osaka, Japan) with a linear hole (6 x 15 mm hole) was placed onto the gold-coated glass plate carrying SAM. Carboxyl groups were activated using $0.1 \mathrm{mM}$ ethyl-3-(3-dimethylaminopropyl)carbodiimide and $0.05 \quad \mathrm{mM} \quad N$-hydroxysuccinimide 
dissolved in an aqueous D-PBS solutions for $15 \mathrm{~min}$ and rinsed with D-PBS two times. A solution of antibody of AFP (clone 1D5) was manually applied onto the linear hole and the coupling reaction was allowed to proceed at RT for $1 \mathrm{~h}$. After the reaction, the surface was rinsed with D-PBS five times and then BSA solution (50 mg/mL in D-PBS) was applied for 30 min to block free activated carboxyl groups. Finally, the plates were rinsed with D-PBS seven times. The antibody-immobilized sensor chips were stored at $4{ }^{\circ} \mathrm{C}$ and used within a week.

Refractive index measurement of solutions

Refractive indexes of D-PBS containing 30, 70, $100 \mathrm{mg} / \mathrm{mL}$ BSA were measured using an Abbe refractometer (DR-A1, Atago Co., Ltd., Tokyo, Japan) at $25^{\circ} \mathrm{C}$.

Effect of BSA concentrations on SPFS-based immunoassay for detection of AFP

AFP solutions (5 ng/mL, $70 \mathrm{pM}$ ) in D-PBS with different BSA concentrations (30, 70, $100 \mathrm{mg} / \mathrm{mL}$ ) were prepared just before each experiment. Anti-human AFP 6D2 antibody labeled with Alexa-Fluor 647 (ahAFP-A647) was added to the AFP solutions at a final concentration of $10 \mathrm{nM}$ and incubated at room temperature for 10 min to form an antibody-AFP complex. The antibody-AFP complex solution was applied to a sensor chip of the SPFS apparatus at a flow rate of $1 \mathrm{~mL} / \mathrm{min}$. Change of fluorescence intensity was 
recorded in real-time by a highly sensitive CCD camera. The same sensor chip was repeatedly used in a series of experiments, that is, the sensor surface was washed by $10 \mathrm{mM}$ glycine- $\mathrm{HCl}$ for 1 minute to remove antibody-AFP complex after each measurement and then antibody-AFP complex solutions containing different concentrations of BSA were applied. Obtained fluorescence intensities were normalized as ratios of the fluorescence intensity of the AFP solution containing $70 \mathrm{mg} / \mathrm{mL}$ BSA.

Quantitative determination of AFP concentrations in fetal bovine serum

A series of AFP solutions (0-20 ng/mL) in FBS were prepared just before each measurement. An aqueous solution containing $35 \mathrm{mg} / \mathrm{mL} \mathrm{NaCl}$, whose refractive index is 1.339, was applied onto a sensor chip to adjust the incident angle to the SPR angle. An ahAFP-A647 solution was added to $2 \mathrm{ml}$ of AFP solution at a final concentration of $10 \mathrm{nM}$ and incubated for 10 min to form the antigen-antibody complex at room temperature. The antigen-antibody complex in FBS was applied to a flow cell of the SPFS apparatus. Fluorescence intensity was recorded. New sensor chips were used in each measurement in a series of AFP solutions in FBS.

Statistical analyses and detection limit

Data from the experiments were expressed as the mean \pm standard deviation (SD). 
Detection limit was determined from the 3.3 SD of baseline measurements (FBS without AFP). All calculation and statistical analysis were executed on R language environment ver. 2.12.0 [17].

\section{Results}

Effects of protein concentration on the intensities of fluorescent light as detected by two SPFS devices with different optical units

Protein concentration in blood samples varies in patients from about 30 to 100 $\mathrm{mg} / \mathrm{mL}[14,15]$. Variations in refractive index due to changes in protein concentration would affect fluorescence intensity detected by SPFS devices. We employed 30, 70, $100 \mathrm{mg} / \mathrm{mL}$ of BSA solutions in D-PBS as a model for patient serums. Refractive indexes of 30, 70, 100 $\mathrm{mg} / \mathrm{mL}$ BSA solutions were 1.339, 1.346 and 1.351, respectively. Reflectance and enhancement factors of the evanescent field relative to the intensity of the incident light were calculated as a function of the incident angle using the Fresnel's equation for the six-layer model (S-LAL10 glass/Cr/Au/SAM/protein adsorbed layer/protein solution) [18]. Fig. 1(a) shows dependences of reflectance and enhancement factors on light incident angles for the 30, 70, $100 \mathrm{mg} / \mathrm{mL}$ BSA solutions. SPR angles, minimums of reflectance, and light incident angles which give maximums of enhancement factors are summarized in Table 1 . The SPR angle and the light incident angle for maxima of enhancement factors increased with the 
increase of BSA concentrations. Although there are small differences between the SPR angle and the angles which give the maximum of the field enhancement factor (Fig. 1(a)), this difference was ignored for SPFS in the following arguments.

SPFS with convergent light beam: Fig. 2(a) shows the alignment of optical units. A parallel incident light beam was focused on the back side of the sensor of the SPFS apparatus. Incident angle range was set to be an angle that gave the maximum enhancement factor $\left(\theta_{\mathrm{MEF}}\right) \pm 5.0^{\circ}$ in the calculation for our SPFS optical unit. The fluorescent chromophore on the sensor surface was irradiated by the enhancement field developed by the incident light with a different incident angle, $\theta_{\mathrm{MEF}} \pm 5.0^{\circ}$. Fluorescent intensity was expected to be proportional to the integration of enhancement field intensity within $\theta_{\mathrm{MEF}} \pm 5.0^{\circ}$. When the center of the incident angle was fixed at the $\theta_{\mathrm{MEF}}$ of $70 \mathrm{mg} / \mathrm{mL} \mathrm{BSA}$, i.e., $58.62^{\circ}$, the relative fluorescent intensities in BSA solutions of 30 and $100 \mathrm{mg} / \mathrm{mL}$ were calculated to be $99.9 \%$ and $99.8 \%$, respectively, as compared to a solution of $70 \mathrm{mg} / \mathrm{mL}$ BSA. Therefore, the relative fluorescent intensity did not depend on protein concentrations.

SPFS with single light beam: Fig. 2(b) shows the alignment of optical units in an instrument with a single light beam. A light beam with small diameter is directed on the back side of the sensor of the SPFS apparatus. When this type of SPFS apparatus is used, an SPR sensory graph is first prepared by plotting the intensity of the reflected light against the incident light angle. Then, the incident angle of the light beam is carefully adjusted to a value 
$0.5^{\circ}$ lower than the SPR angle [11] for each sample and then the fluorescent intensity is determined at that angle. In a clinical setting, however, this is a time consuming and tedious step. An SPFS apparatus with a fixed incident angle of the light beam should be employed. In the following calculation, the incident light is fixed at the angle which is optimal for 70 $\mathrm{mg} / \mathrm{mL}$ BSA solution, $58.62^{\circ}$. Changing the BSA concentration to 30 and $100 \mathrm{mg} / \mathrm{mL}$ led to slight decreases in the field enhancement factor of $93.3 \%$ and $94.7 \%$, respectively, of that observed for a solution of $70 \mathrm{mg} / \mathrm{mL}$ BSA. Fluorescent light intensity is expected to be proportional to the intensity of the evanescent field. Therefore, this calculation indicates that the fluorescence intensity measured by the SPFS apparatus with single light beam is dependent on the protein concentration in the blood of patients.

Fluorescent light intensities estimated for SPFS devices with different optical systems are also summarized in Table 1 for ease of comparison. These calculations indicated that the SPFS apparatus with convergent optical system efficiently suppressed the dependency of the fluorescence intensity on the protein concentrations in the serum in patients and thus is suitable for POC.

SPFS apparatus with convergent optical system

Our SPFS apparatus with the convergent optical system was composed of an light source, alignment of optical units, SPR measurement and SPFS measurement units as 
schematically shown in Fig. 2(a). A photograph of its prototype is shown in Fig. 3(a). Light from a pointed LED was passed through an iris, collimated, p-polarized, and then focused on the back side of a sensor, giving a fixed range (center $\pm 5^{\circ}$ ) of incident angle. Reflected light was captured by a CCD camera for SPR measurement and the fluorescence image on the sensor surface was observed by a highly sensitive CCD camera for SPFS measurement.

An image of the reflected light is shown in Fig. 3(b-1) and a line profile of brightness is plotted as a function of pixel position in a horizontal direction, i.e., by varying the incident angle, in Fig. 3(b-2). Horizontal movement of the darker area reflects the change of the reflective index on the sensor surface. The SPR angle was determined from this line profile as a pixel position at which light intensity displayed the smallest value, as indicated by a dotted line. Fig. 3(c-1) also included an SPFS image obtained by a highly sensitive CCD camera during the flow of the AFP-antibody-fluorescent chromophore complex over a sensor chip functionalized with primary anti-AFP antibody. SPFS images were sectioned into 26 small areas with $100 \times 100$ pixels. Integrated intensities of fluorescent light in each area were calculated with home-made analysis software and are plotted with time in Fig. 3(c-2). Averaged whole area fluorescent intensity was employed hereafter.

Quantitative measurement of AFP in fetal bovine serum

The efficacy of our SPFS system for detection of low levels of AFP in serum was 
evaluated by using solutions of AFP in FBS. Anti-human AFP 6D2 antibody labeled with Alexa-Fluor 647 was mixed with AFP solution in FBS and incubated at room temperature for 10 min to form an AFP antibody-Alexa-Fluor 647 complex. The solution was applied to a sensor surface on which primary anti-AFP antibody was immobilized. The intensities of fluorescent light were integrated for 26 small areas (Fig. 3(c-1)) before and after the AFP antibody-Alexa-Fluor 647 complexes were applied. Fluorescent light intensity caused by the complex on the sensor surface was estimated by the difference between the integrated light intensity before and after the complex was applied. As shown Fig. 4(a), the fluorescent light intensity from the complex on the sensor surface linearly increased with time, indicating that the antibody-AFP complex was bound to the primary antibody immobilized on the sensor chip. Larger increments of fluorescence intensity were observed with increasing AFP concentrations. The elevations in the rates of fluorescence intensities were used to rapidly determine AFP concentration in solutions. The slopes of the lines, corresponding to changes in fluorescent intensity as a function of time, were plotted against the AFP concentrations (Fig. 4(b)). Linear regression analysis of these plots (Fig. 4(b)) indicated a strong linear relationship $\left(\mathrm{R}^{2}=0.995\right)$. The standard derivation of the elevation in the rates of fluorescence intensity obtained from FBS without AFP was 338 a.u./min $(n=12)$. Therefore the limit of detection of our prototype SPFS-convergent apparatus was estimated to be $0.68 \mathrm{ng} / \mathrm{mL}$ for AFP. 
Effect of protein concentration on fluorescence intensity

As shown in Table 1, we previously examined the effect of protein concentration on fluorescence intensity in the SPFS apparatus with the convergent optical unit using Fresnel's equation. The incident angle was adjusted to be optimal for detecting AFP in $70 \mathrm{mg} / \mathrm{mL}$ BSA. Mixtures of anti-human AFP antibody labeled with Alexa-Fluor 647 and 70 pM AFP in solutions containing 30,70 , or $100 \mathrm{mg} / \mathrm{mL}$ BSA were applied to the sensor surface. The fluorescent light intensity linearly increased with time (Fig. 5). The rates of the elevations of fluorescence intensity varied with BSA concentrations. Relative elevation rates for 30 and $100 \mathrm{mg} / \mathrm{mL}$ BSA/DPBS solutions were 0.97 and 0.92, respectively, for the convergent light SPFS apparatus.

\section{Discussion}

We assessed the efficacy of our SPFS apparatus with a convergent optical system as

a POC device with high sensitivity. Immunoassay of AFP using our SPFS apparatus demonstrated that the fluorescent intensity increased linearly due to binding of AFP-antibody-Alexa-Fluor 647 complex to a sensor surface and the slope was in proportion to the AFP concentrations (Fig. 4(b)). Our SPFS-based immunoassay could determine the AFP concentration within 20 min after we received the plasma sample. The limit of detection 
for AFP in FBS was determined to be $0.68 \mathrm{ng} / \mathrm{mL}$. There are several sensitive assay methods used in clinical diagnosis, such as enzyme-linked immunosorbent assays (ELISA), chemiluminescence immune assay (CLIA), and radioimmunoassay (RIA). The detection limit of AFP is about $2 \mathrm{ng} / \mathrm{mL}$ for commercially available ELISA [19], $0.5 \mathrm{ng} / \mathrm{mL}$ for ECLIA [20], and RIA [21]. Approximately 97\% of the healthy subjects have AFP levels less than 8.5 ng/mL [22]. We therefore concluded that our prototype SPFS apparatus is sensitive enough for clinical diagnosis of AFP concentrations and gives clinical test results within the permissive range of time for measurements at the bed-side. ELISA, CLIA, and RIA, all of which are clinically used, require more than 10 steps including washing samples to remove unbound complexes. In contrast, our SPFS-based immunoassay could determine AFP concentration in samples without removal of unbound complexes (Fig. 4). This result makes this SPFS apparatus suitable for POC testing, eliminating the time consuming and tedious procedures and allowing immediate clinical management decisions to be made.

The application of this SPFS apparatus to clinical diagnosis will benefit from some improvements. The SPFS apparatus utilizes the enhanced evanescent field caused by the SPR phenomenon of excitation of a fluorescent dye. As shown in Fig. 1, the light incident angle, at which the evanescent field gives its maximum response, depends on the refractive index near the metal surface. Therefore, fluorescence intensity changed in a manner dependent on the protein concentration of the sample, even though the concentration of the antigen AFP 
remained the same. We developed the SPFS apparatus with the convergent optical system to solve this problem and expected that the incident light angle would equal the SPR angle $\pm 5^{\circ}$. Theoretical calculation using Fresnel's equation and experiments demonstrated less variation in the changes in fluorescence intensity caused by differences in protein concentration. Although the SPFS-convergent apparatus greatly suppressed the effect of BSA concentration on the relative increases in the rates of fluorescence increase (Fig. 5), these dependences were larger than those calculated using Fresnel's equation. We suspect non-uniformity of the incident light might be the cause of the difference between the experimental and calculated values. Ideally, the intensity of the incident light should be the same at any incident angle. A LED was used as a light source in our SPFS apparatus and the emitted light whose directivity was larger than $10^{\circ}$ was cut by an iris to obtained uniform light flux. As seen in the SPFS image shown in Fig. 3(c-1), however, its central section was brighter than its periphery. Thus, the illumination assembly needs to be improved to reduce the size of illuminated area. Additionally, the use of a photomultiplier instead of CCD camera might improve readability of signal by permitting the collection all fluorescence light emitted from the sample.

Many bright spots were observed in SPFS image of the sensor surface as shown in Fig. 3(c-1). Their size was larger than $10 \mu \mathrm{m}$ in diameter. When a gold surface was observed by an optical microscope and an atomic force microscope, such large granules were not found. These bright spots might be caused by coupling of surface plasmon-polaritons due to the 
roughness of the granules of gold on a sensor chip, as pointed out by Rothenhaesler [23]. If number and location of these spots could be well controlled, fluorescent intensities would be greater enhanced due to the effect of localized plasmon-polaritons. These bright spots, however, caused heterogeneity of the sensor-chip surface, making difficult the direct correlation between fluorescent intensity and antigen AFP concentration.

\section{Conclusions}

We fabricated an SPFS apparatus using a convergent optical system, giving a fixed range or incident light. Both theoretical calculation and immunoassay of AFP demonstrated that our SPFS apparatus was relatively robust against fluctuations of refractive indexes of specimens. This apparatus could determine concentrations of AFP in FBS quantitatively under the concentration of clinical cut-off of AFP. Our results will contribute to the development of small, simple but highly sensitive and precise measurement devices.

\section{Acknowledgments}

We thank Mr. Noriaki Toda for his technical support. This study was supported by the Development of Systems and Technology for Advanced Measurement and Analysis, assigned by the Japan Science and Technology Agency (JST). 
References

1. Price CP: Point of care testing. BMJ 322:1285-1288, 2001.

2. Lonnberg M, Carlsson J: Membrane assisted isoform immunoassay. A rapid method for the separation and determination of protein isoforms in an integrated immunoassay. $\mathrm{J}$ Immunol Methods 246:25-36, 2000.

3. Malhotra R, Patel V, Vaqu JP, Gutkind JS, Rusling JF: Ultrasensitive Electrochemical Immunosensor for Oral Cancer Biomarker IL-6 Using Carbon Nanotube Forest Electrodes and Multilabel Amplification. Anal Chem 82:3118-3123, 2010.

4. Bolduc OR, Clouthier CM, Pelletier JN, Masson J: Peptide self-assembled monolayers for label-free and unamplified surface plasmon resonance biosensing in crude cell lysate. Anal Chem 81:6779-6788, 2009.

5. Bolduc OR, Pelletier JN, Masson J: SPR Biosensing in crude serum using ultralow fouling binary patterned peptide SAM. Anal Chem 82:3699-3706, 2010.

6. Teramura Y, Arima Y, Iwata H: Surface plasmon resonance-based highly sensitive immunosensing for brain natriuretic peptide using nanobeads for signal amplification. Anal Biochem 357:208-215, 2006.

7. Yuk JS, MacCraith BD, McDonagh C: Signal enhancement of surface plasmon-coupled emission (SPCE) with the evanescent field of surface plasmons on a bimetallic paraboloid biochip. Biosens Bioelectron 26:3213-3218, 2011. 
8. Hosokawa K, Omata M, Maeda M: Immunoassay on a power-free microchip with laminar flow-assisted dendritic amplification. Anal Chem 79:6000-6004, 2007.

9. Ikami M, Kawakami A, Kakuta M, Okamoto Y, Kaji N, Tokeshi M et al: Immuno-pillar chip: a new platform for rapid and easy-to-use immunoassay.. Lab Chip 10:3335-3340, 2010.

10. Liebermann T, Knoll W: Surface-plasmon field-enhanced fluorescence spectroscopy. Colloids and Surfaces A Physicochemical and Engineering Aspects 171:115-130, 2000.

11. Murakami T, Arima Y, Toda M, Takiguchi H, Iwata H: Effect of dielectric spacer thickness on signal intensity of surface plasmon field-enhanced fluorescence spectroscopy. Anal Biochem 421(2):632-639, 2012

12. Yu F, Persson B, Löfås S, Knoll W: Surface plasmon fluorescence immunoassay of free prostate-specific antigen in human plasma at the femtomolar level. Anal Chem 76:6765-6770, 2004.

13. Arima Y, Teramura Y, Takiguchi H, Kawano K, Kotera H, Iwata H. Surface plasmon resonance and surface plasmon field-enhanced fluorescence spectroscopy for sensitive detection of tumor markers. In: Rasooly A, Herold KE, editors. Methods in Molecular Biology 503: Biosensors and Biodetection. Methods Mol Biol 503:3-20; 2009. p. 3-20

14. Rinsho Kensaho Teiyou (Kanai's manual of clinical laboratory medicine), 33rd ed. Kanehara Shuppan, 2010 
15. Wallach JD: Interpretation of diagnostic tests, 7th Ed. Lippincott Williams \& Wilkins, 2000.

16. Toda M, Arima Y, Iwata H: Complement activation on degraded polyethylene glycol-covered surface. Acta Biomater 6:2642-2649, 2010.

17. R Development Core Team: R: A Language and Environment for Statistical Computing. R Foundation for Statistical Computing, 2009.

18. W. Knoll, Polymer thin films and interfaces characterized with evanescent light, Makromol. Chem. 192 (1991) 2827-2856.

19. AFP (Human) ELISA Kit Datasheet [Internet]. Taiwan: Abnova Corp.; @2014 [cited 2014 Mar 03]. Available from:

http://www.abnova.com/products/products_detail.asp?catalog_id=KA0202

20. Blackburn GF, Shah HP, Kenten JH, Leland J, Kamin RA, Link J et al:

Electrochemiluminescence detection for development of immunoassays and DNA probe assays for clinical diagnostics. Clin Chem 37:1534-1539, 1991.

21. Protocol of Alpha-Foetoprotein ELSA [Internet]. Codolet, France: Cisbio Bioassay. (C)2014 [cited 2014 Mar 03]. Available from: http://www.ria-cis.com/sites/default/files/ressources/02-GB-ELSA2-AFP-Mod25.pdf

22. Ball D, Rose E, Alpert E: Alpha-fetoprotein levels in normal adults. Am J Med Sci 303:157-159, 1992. 
23. Rothenhaeusler B, Knoll W: Surface-plasmon microscopy. Nature 332:615-617, 1988. 


\section{Footnotes}

1 Abbreviations used: AFP, $\alpha$-fetoprotein; a.u., arbitrary unit; BSA, bovine serum albumin;CCD, charge coupled device; D-PBS, Dulbecco's phosphate-buffered saline;EDC, 1-ethyl-3-(3-dimethylaminopropyl) carbodiimide hydrochloride; FBS, fetal bovine serum; f-PSA, free prostate specific antigen; LED, light-emitting diodes; ND, neutral density; PC, personal computer; PMMA, poly(methylmethacrylate); POC, point-of-care; POCT, point-of-care testing; RT, room temperature; SD, standard deviation; SPFS, surface plasmon field-enhanced fluorescence spectroscopy; SPR, surface plasmon resonance. 
Figure captions

Scheme 1. Schematic illustration of SPFS-based immunoassay for the detection of AFP. (a)

The primary antibody was immobilized on the sensor surface; (b) AFP-antibody complex was

flowed over the sensor surface; (c) The primary antibodies captured the AFP complexes and a fluorescence detector (CCD camera) captured fluorescent image used by the AFP complexes bound on the sensor surface.

Fig 1. Calculation of reflectance and enhancement factors of the evanescent field relative to the intensity of the incident light as a function of the incident angle using the Fresnel's equation for the six-layer model (S-LAL10 glass/Cr/Au/SAM/protein adsorbed layer/protein solution). The calculation was performed in the presence of BSA solutions with different concentrations: - - - -; $30 \mathrm{mg} / \mathrm{mL}$ (refractive index, $n=1.3389), \longrightarrow ; 70 \mathrm{mg} / \mathrm{mL}(n=$ $1.3456), \cdots \cdots ; 100 \mathrm{mg} / \mathrm{mL}(n=1.3505)$. SPFS apparatus with a convergent light beam detects fluorescence at a fixed range of the incident angle (shaded area) while SPFS device with a single light beam detects fluorescence at a fixed incident angle (solid line).

Fig. 2. Two kinds of SPFS devices. (a) SPFS device with a convergent light beam. The light was passed through an iris (IH-08R, Sigma Koki, Tokyo, Japan) to cut the light of which 
directivity was larger than $5^{\circ}$, paralleled and then the parallel light was converged on the back side of the sensor. (b) SPFS device with a single light beam. A parallel single beam with small diameter is directly irradiated on the back side of the sensor.

Fig. 3. SPFS device with a convergent light beam. (a) Photo of a prototype device; (b-1) CCD image of reflected light; (b-2) Line profile of the CCD image. (c-1) Fluorescent image obtained by the SPFS device with a convergent light beam during flowing of AFP-antibody-Alexa-Fluor 647 complex (AFP: $5 \mathrm{ng} / \mathrm{mL}$ ). Yellow boxes indicate regions at which light intensity are measured. (c-2) Changes of fluorescence intensity at each region shown in panel c-1 during flowing of AFP-antibody-Alexa-Fluor 647 complex (AFP: 5 ng/mL) over a sensor chip functionalized with primary anti-AFP antibody.

Fig. 4. Detection of AFP by an SPFS device with a convergent light system. (a); Fluorescence intensity changes during the flow of FBS solutions containing ahAFP-A647-AFP with the indicated AFP concentrations over a sensor chip of a SPFS device with a convergent light system. Lines represent linear fittings of data points to calculated slopes of the increases in fluorescence intensity over time $\left(\mathrm{R}^{2}=0.995\right)$. (b): Slopes of increases of fluorescence intensity as a function of time are plotted against AFP concentrations. Dot line shows means + 3.3SD in the absence of AFP. 
Fig. 5. Changes in fluorescence intensity during flowing of hAFP-A647-AFP complexes.

AFP (5 ng/ml) in solution with different SPA concentrations detected by SPFS with a convergent light system. BSA concentration; 30( $\diamond), 70(\bigcirc), 100(\triangle) \mathrm{mg} / \mathrm{mL}$. 
Table 1. Summary of theoretical calculations for immunoassay detected using SPFS apparatus with single light beam and with convergent optical system.

\begin{tabular}{|c|c|c|c|}
\hline & \multicolumn{3}{|c|}{ Bovine serum albumin concentration, $\mathrm{mg} / \mathrm{ml}$} \\
\hline & 30 & 70 & 100 \\
\hline Refractive index & 1.339 & 1.346 & 1.351 \\
\hline SPR angle & 58.68 & 59.15 & 59.51 \\
\hline $\begin{array}{l}\text { Angle gives maximum enhancement } \\
\text { factor }\end{array}$ & 58.15 & 58.62 & 58.97 \\
\hline $\begin{array}{l}\text { Enhancement factor at the angle optimal } \\
\text { for } 70 \mathrm{mg} / \mathrm{mL} \mathrm{BSA}\end{array}$ & & & \\
\hline SPFS with convergent light beam & 5.730 & 5.736 & 5.726 \\
\hline SPFS with single light beam & 13.26 & 14.21 & 13.45 \\
\hline
\end{tabular}


(a)

(b)

(c)

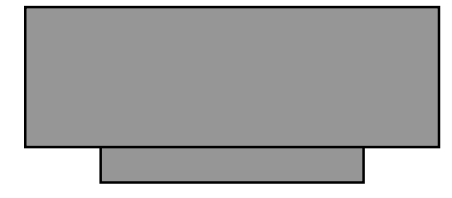

Detector for fluorescence
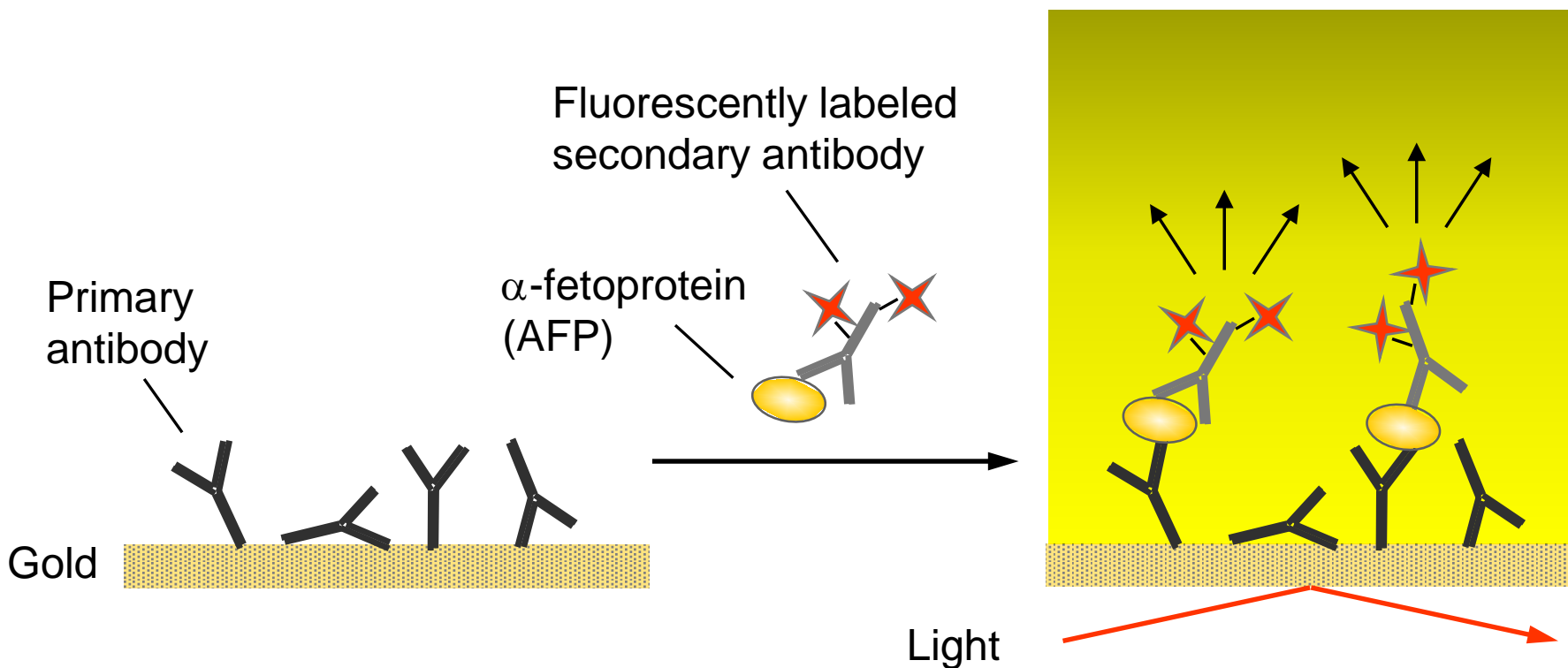

$\mathrm{Lz}=190 \mathrm{~nm}$

Light

Scheme 1 


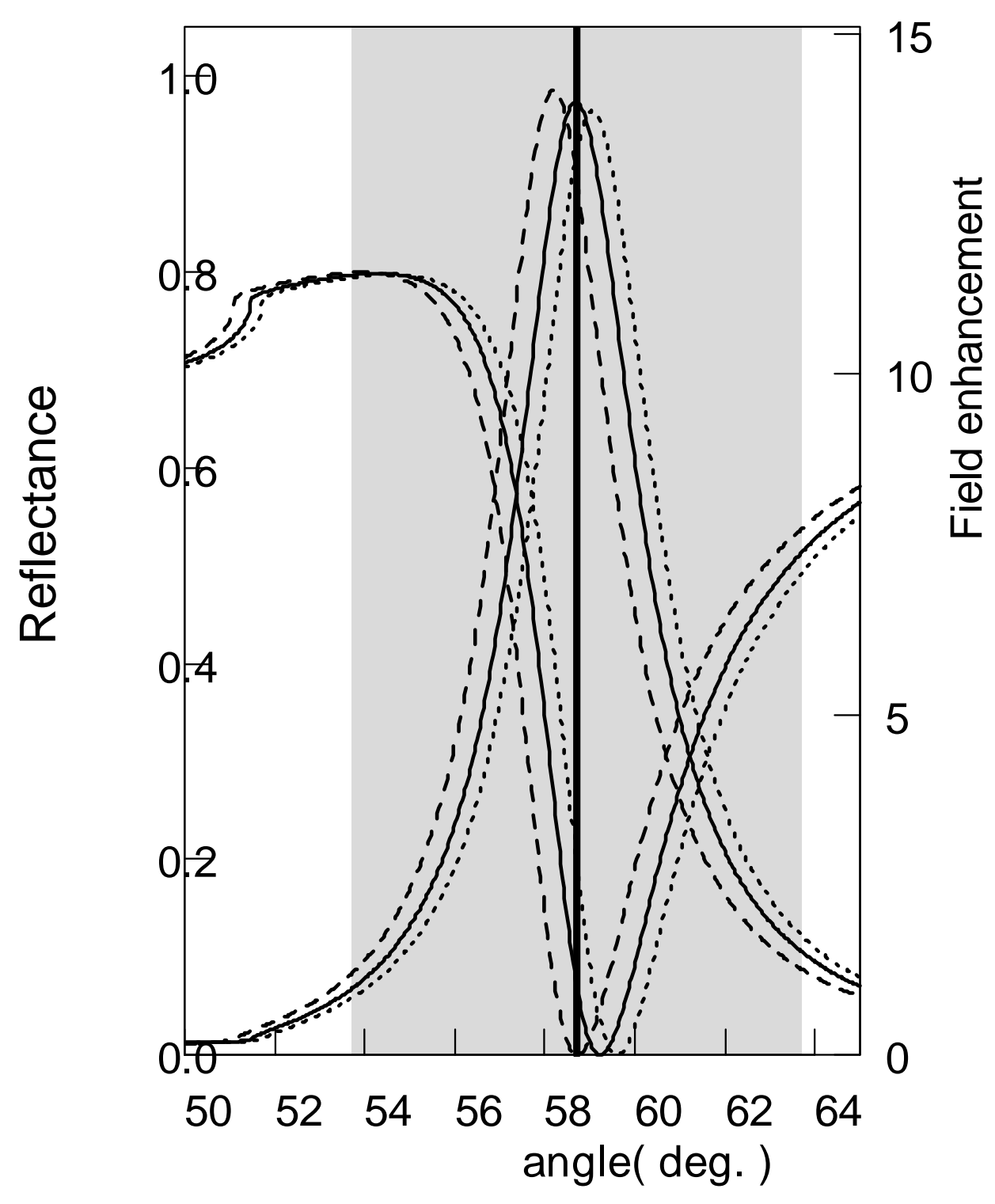

Fig. 1 
(a)

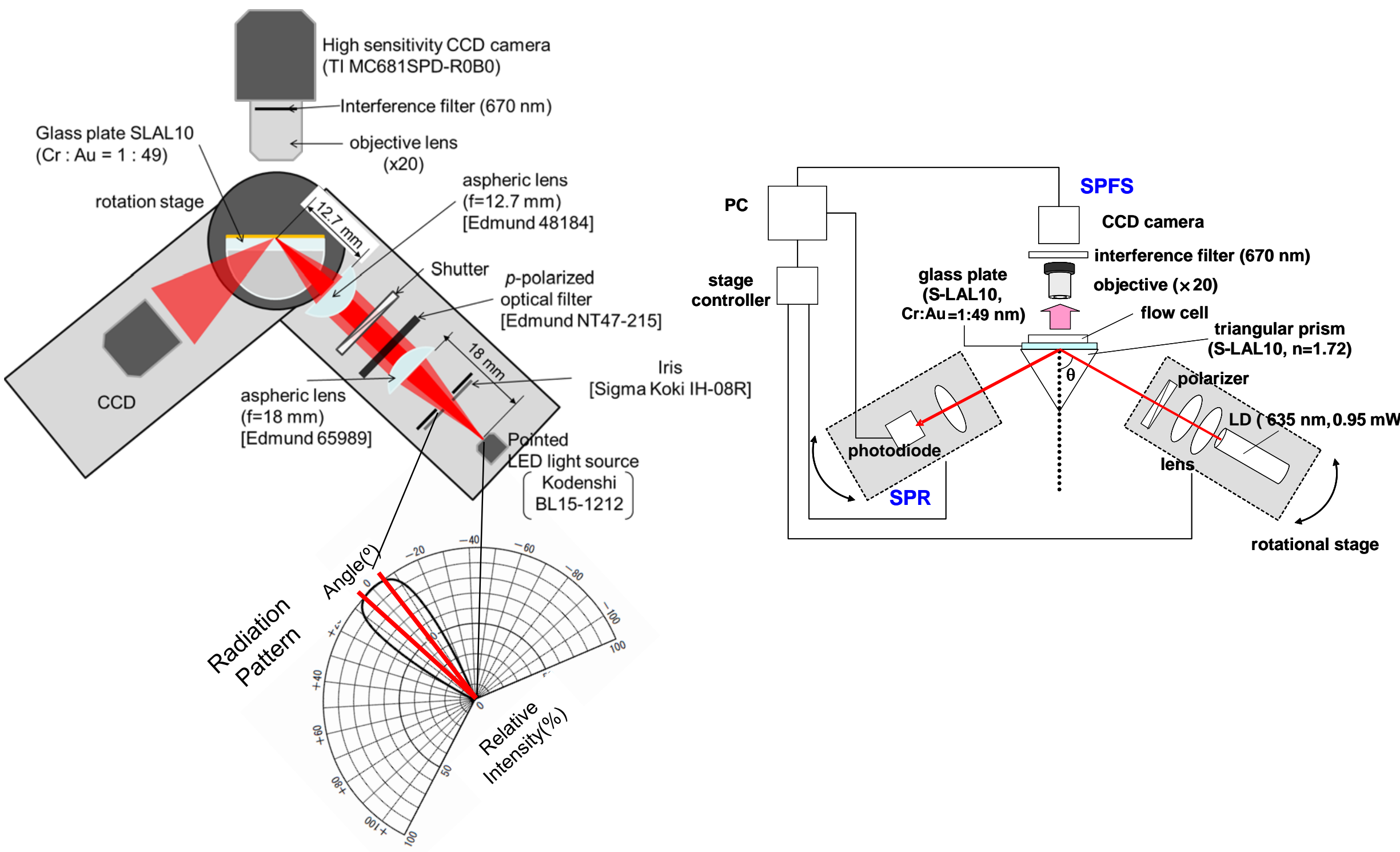

(b)

Fig. 2 


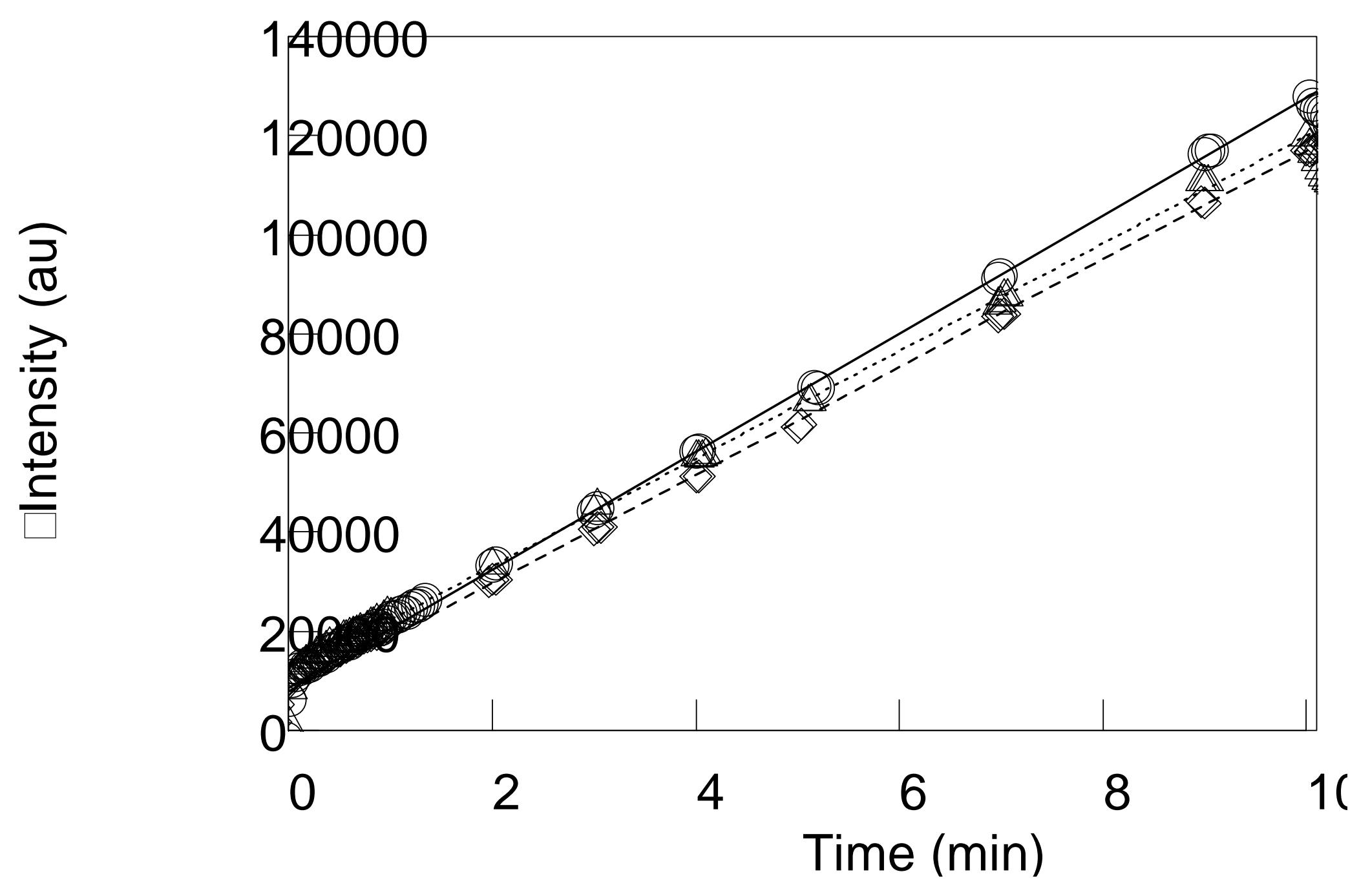

Fig. 5 